\title{
Detection of Damage in Rail Head by Using SAFE Method
}

\author{
Sara.Teidj, Abdellah. Driouach, Abdellatif. Khamlichi \\ Systems of Communications and Detection Laboratory \\ Faculty of Sciences, Tetuan, Morocco \\ sara.teidj@gmail.com, adrioauch@hotmail.com, khamlichi7@yahoo.es
}

\begin{abstract}
The first cause of train derailment incidents is due to the transverse defect which occurs in the railhead. It is typically an open or internal crack developing in a plane which is generally perpendicular to the direction of the rail. The most method used today of rail inspection is based on ultrasound .The Ultrasounds based testing is performed according to the excitation-echo procedure. It is conducted conventionally by using a contact excitation probe that rolls on the rail head or by a contact-less system using laser as excitation and air coupled acoustic sensors for wave reception [1].

In this work, Propagation of guided elastic waves in a rail has been modelled by using the semi-analytical finite element method [2]. We have demonstrated the existence of several frequency windows that can be used to excite propagating modes with high deformation in the railhead.
\end{abstract}

Keywords-rail; waveguide; pulsed laser; air-coupled; safe method; laser vibrometry.

\section{Introduction}

With increasing railways traffic and augmenting speed of modern trains, maintenance of rails constitutes a major concern of safety for this form of transportation. The objective is to impede catastrophic failures that can threaten human lives and provoke huge economic loss [3].

Rail is the major cause of train accidents [4]. Almost $70 \%$ of the causes of train derailments are attributed to the transverse defect appearing in the railhead [5]. Current inspection techniques are mainly based on electromagnetic induction [6] or ultrasound [7].

Since an analytical method cannot be used to modeling wave propagation in waveguides of any arbitrary cross-sections a new numerical method has been developed. It is the Semi-Analytical Finite Element (SAFE) method [8]. This method has been successfully used for several structures and in particular in the case of any cross-section beams such as rails. The method requires the finite element discretization of the cross- section of the waveguide, and assumes harmonic motion along the wave propagation direction.

We present the SAFE method and we will apply it through the Comsol Mutiphysics software to model the elastic waveguides in a rail. We illustrate the study of propagative modes that can account for the presence of transverse defects in the railhead. 


\section{Elastic Waveguides in a Rail}

\subsection{Propagation of elastic waveguides}

Let us consider an elastic waveguide with waves propagating along the direction $x_{3}$ with the wave number $k$ and frequency $\omega$. The cross-section of the waveguide is in the plane $\left(O, x_{1}, x_{2}\right)$.

The equation of motion is written:

$$
C_{i p l m} \frac{\partial^{2} u_{l}}{\partial x_{p} \partial x_{m}}=\rho \frac{\partial^{2} u_{i}}{\partial t^{2}} \quad i, p, l, m=1,2,3
$$

Where $C_{i p l m}$ are the terms of the elasticity tensor, $\rho$ the density of the material, $u_{i}$ the components of the displacement vector and $t$ the time.

In harmonic mode the general solution of the waveguide is in the form

$$
u_{i}\left(x_{1}, x_{2}, x_{3}, t\right)=U_{i}\left(x_{1}, x_{2}\right) \exp \left[j\left(k x_{3}-\omega t\right)\right] i=1,2,3
$$

Where $k$ the wave number, $\omega$ pulsation and $U_{i}$ amplitude.

By substituting equation (2) in equation (1), it comes

$$
C_{i p l m} \frac{\partial^{2} u_{l}}{\partial x_{p} \partial x_{m}}+\rho \omega^{2} u_{i}=0 \quad i, p, l, m \in\{1,2,3\}
$$

By explaining the calculation of derivation with respect to $x_{3}$ we obtain:

$$
C_{i p l m} \frac{\partial^{2} U_{l}}{\partial x_{p} \partial x_{m}}+j k\left(C_{i 3 l p}+C_{i p l 3}\right) \frac{\partial U_{l}}{\partial x_{p}}-k^{2} C_{i 3 l 3} U_{l}+\rho \omega^{2} \delta_{i l} U_{l}=0
$$

with

$$
i, l \in\{1,2,3\} \quad p, m \in\{1,2\}
$$

To equations (4), we must add the boundary conditions on the free boundary of the right section which are written:

$$
T_{i}=\sigma_{i p} n_{p}=C_{i p l m} \frac{\partial U_{l}}{\partial x_{m}} n_{p}+j k C_{i p l 3} U_{l} n_{p}=0
$$

The phase velocity is defined by:

$$
c_{p}=\frac{\omega}{k}
$$

Where one takes the real wave numbers knowing that in general they are complex.

If the wave number is pure imaginary, the wave is said to be evanescent and does not propagate.

When $\operatorname{real}(k) \neq 0$, the propagation is possible, then the imaginary part $\operatorname{imag}(k)$ introduces attenuation and the wave is damped. 


\subsection{Calculate the Dispersion curves in a rail by Comsol Multiphysics software}

We present a method which makes it possible to use Comsol Multiphysics software to perform the calculation of dispersion curves. This method uses the PDE mode (Partial Differential Equation), which allows to the user to define the problem by writing the differential equations to the partial differentials that govern it.

The PDE coefficient form interface of Comsol Multiphysics makes it possible to solve a boundary problem governed by the system of differential equations with partial derivatives having the following [9]:

$$
\begin{array}{lr}
\nabla \cdot(c \nabla \vec{u}+\alpha \vec{u})-\beta \nabla \vec{u}-a \vec{u}+\lambda d_{a} \vec{u}=0 & \text { dans } \Omega \\
\vec{n} \cdot(c \nabla \vec{u}+\alpha \vec{u})=0 & \text { sur } \partial \Omega
\end{array}
$$

where $\vec{n}$ the unit normal vector external to the domain and $\vec{u}$ the unknown field.

A reformulation of the SAFE problem defined by the system of equations (4) has been proposed by Predoi [10]. Equation (7) can be rewritten in the following explicit form:

$$
C_{i p l m} \frac{\partial^{2} u_{p}}{\partial x_{l} \partial x_{m}}+\left(\alpha_{i p l}-\beta_{i p l}\right) \frac{\partial u_{p}}{\partial x_{l}}-a_{p} u_{p}+\lambda d_{i p} u_{p}=0
$$

Similarly the Neumann condition defined by equation (8) is identified by

$$
C_{i p l m} \frac{\partial u_{p}}{\partial x_{m}} n_{l}+\alpha_{i p l} u_{p} n_{l}=0
$$

In order to rewrite the system of equations (9) and (10) the set of the following matrices is introduced:

$$
\begin{array}{r}
d_{a}=\left[\begin{array}{cc}
O & D \\
M & O
\end{array}\right] ; c=\left[\begin{array}{cc}
C & O \\
O & O
\end{array}\right] ; \alpha=\left[\begin{array}{cc}
O & j A \\
O & O
\end{array}\right] \\
\beta=\left[\begin{array}{cc}
O & -j B \\
O & O
\end{array}\right] ; \alpha=\left[\begin{array}{cc}
M & O \\
O & M
\end{array}\right]
\end{array}
$$

The submatrices are given by

$$
\begin{gathered}
A=\left[\begin{array}{lll}
C_{15} & C_{14} & C_{13} \\
C_{65} & C_{64} & C_{63} \\
C_{65} & C_{64} & C_{63} \\
C_{25} & C_{24} & C_{23} \\
C_{55} & C_{54} & C_{53} \\
C_{45} & C_{44} & C_{43}
\end{array}\right] ; B=\left[\begin{array}{lll}
C_{51} & C_{56} & C_{55} \\
C_{56} & C_{52} & C_{54} \\
C_{41} & C_{46} & C_{45} \\
C_{46} & C_{42} & C_{44} \\
C_{31} & C_{36} & C_{35} \\
C_{36} & C_{32} & C_{34}
\end{array}\right] \\
C=\left[\begin{array}{llllll}
C_{11} & C_{16} & C_{16} & C_{12} & C_{15} & C_{14} \\
C_{61} & C_{66} & C_{66} & C_{62} & C_{65} & C_{64} \\
C_{61} & C_{66} & C_{66} & C_{62} & C_{65} & C_{64} \\
C_{21} & C_{26} & C_{26} & C_{22} & C_{25} & C_{24} \\
C_{51} & C_{56} & C_{56} & C_{52} & C_{55} & C_{54} \\
C_{41} & C_{46} & C_{46} & C_{42} & C_{45} & C_{44}
\end{array}\right]
\end{gathered}
$$




$$
\begin{gathered}
D=\left[\begin{array}{ccc}
-C_{55} & -C_{54} & -C_{53} \\
-C_{45} & -C_{44} & -C_{43} \\
-C_{35} & -C_{34} & -C_{33}
\end{array}\right] ; \\
M=\left[\begin{array}{ccc}
-\rho \omega^{2} & 0 & 0 \\
0 & -\rho \omega^{2} & 0 \\
0 & 0 & -\rho \omega^{2}
\end{array}\right] ; O=\left[\begin{array}{lll}
0 & 0 & 0 \\
0 & 0 & 0 \\
0 & 0 & 0
\end{array}\right]
\end{gathered}
$$

Where the $C_{p m} \quad p, m=1, \ldots, 6$ are the contracted coefficients of the elasticity tensor and $\lambda=k$.

In the case of a linear and isotropic elastic material of Young's modulus $E$ and Poisson's ratio $v$, the matrices $A, B, C$ and $D$ are written

$$
\begin{aligned}
& A=\frac{E}{(1+v)(1-2 v)}\left[\begin{array}{ccc}
0 & 0 & v \\
0 & 0 & 0 \\
0 & 0 & 0 \\
0 & 0 & 1-v \\
1-2 v & 0 & 0 \\
0 & 1-2 v & 0
\end{array}\right] \\
& B=\frac{E}{(1+v)(1-2 v)}\left[\begin{array}{ccc}
0 & 0 & 1-2 v \\
0 & 0 & 0 \\
0 & 0 & 0 \\
0 & 0 & 1-2 v \\
v & 0 & 0 \\
0 & v & 0
\end{array}\right] \\
& C=\frac{\mathbf{5}}{(1+v)(1-2 v)}\left[\begin{array}{cccccc}
1-v & 0 & 0 & v & 0 & 0 \\
0 & 1-2 v & 1-2 v & 0 & 0 & 0 \\
0 & 1-2 v & 1-2 v & 0 & 0 & 0 \\
v & 0 & 0 & 1-v & 0 & 0 \\
0 & 0 & 0 & 0 & 1-2 v & 0 \\
0 & 0 & 0 & 0 & 0 & 1-2 v
\end{array}\right] \\
& D=\frac{E}{(1+v)(1-2 v)}\left[\begin{array}{ccc}
2 v-1 & 0 & 0 \\
0 & 2 v-1 & 0 \\
0 & 0 & 2 v-1
\end{array}\right]
\end{aligned}
$$

\section{Results and discussion}

We apply the SAFE approach to determining the dispersion curves in the case of a rail. The knowledge of dispersion curves presents a great interest in the domain of the CND which concerns frequencies up to $50 \mathrm{kHz}[11]$.

We consider a homogeneous and isotropic rail whose material properties are: $\rho=7800 \mathrm{~kg} / \mathrm{m}^{3}$; $E=2 \times 10^{11} \mathrm{~Pa}$ and $v=0.3$

We consider a transverse defect which is modeled by a loss of section in the form of a rectangle whose dimensions are: $0.01 \mathrm{~m} \times 0.02 \mathrm{~m}$. 
Sara.Teidj, Abdellah. Driouach, Abdellatif. Khamlichi. Detection of Damage in Rail Head by Using SAFE Method. Case of Information Systems Management of a Private Moroccan University. Transactions on Machine Learning and Artificial Intelligence, Vol 5 No 4 August (2017); p: 614-621

Figure 1 and 2 show the section of the rail without defect and the rail with defect.
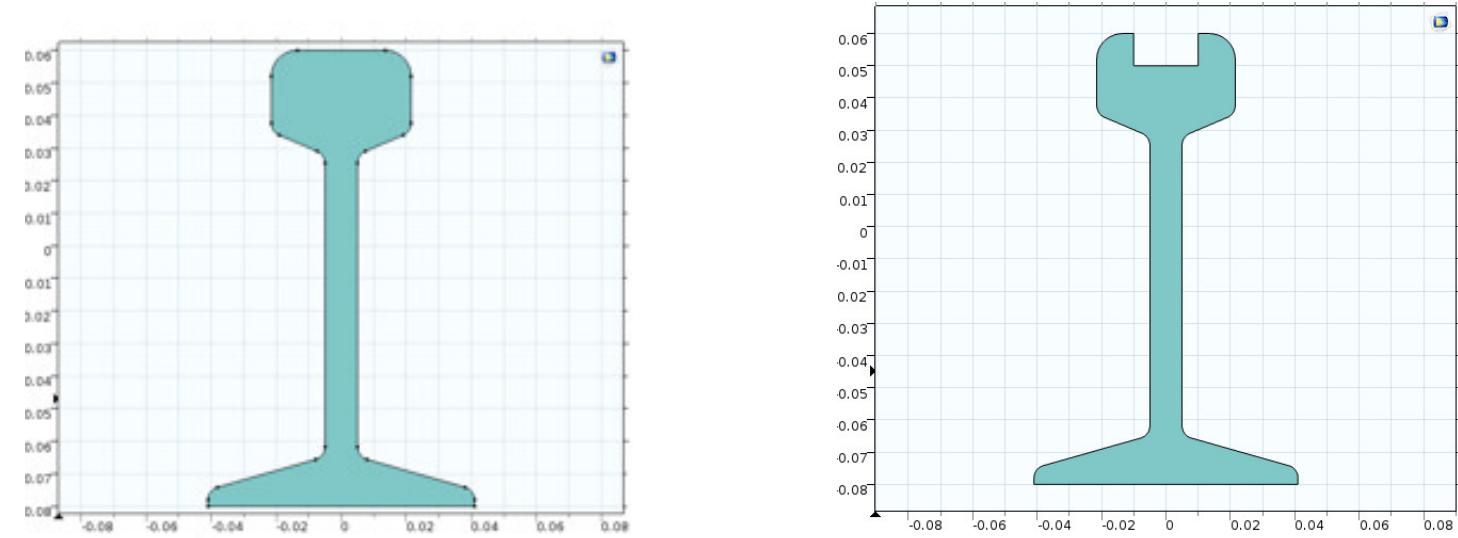

Figure 2: European Rail Geometry Vignole; with defect in railhead

Figure 3 and 4 show the mesh used in the SAFE method for the rail without defect and the rail with defect.

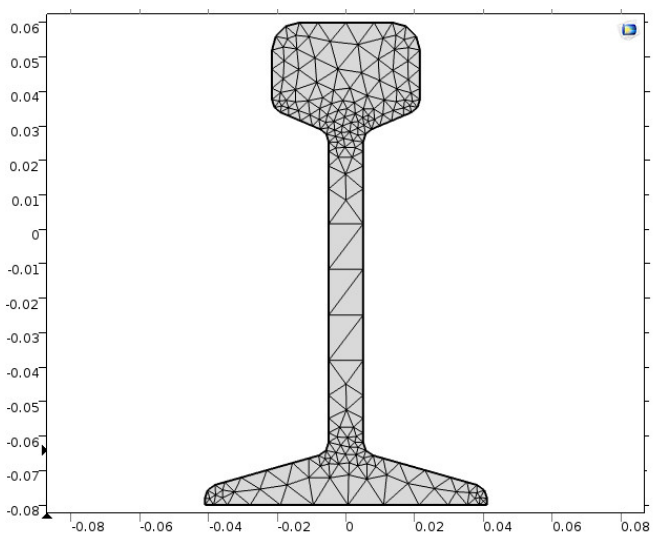

Figure 3: Meshing of European rail Vignole UIC 18.3

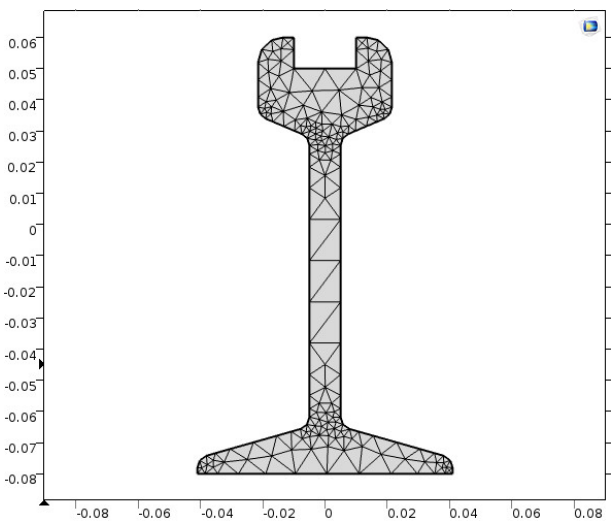

Figure 4: Meshing of European rail Vignole UIC 18.3with defect in railhead

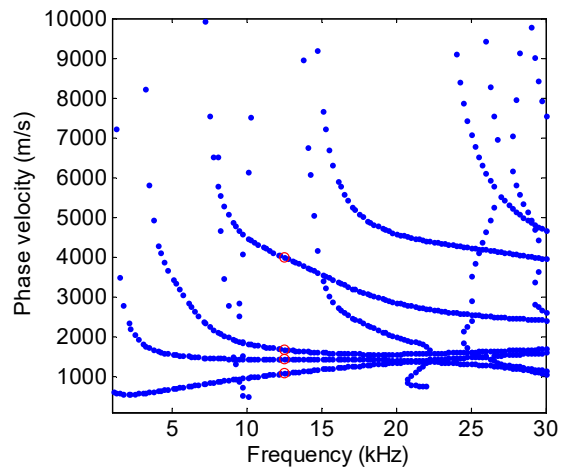

Figure 5: Dispersion curves in terms of phase velocity for the intact rail; the points red are for $12.5 \mathrm{kHz}$ 
The dispersion curves in terms of phase velocity are shown in figure 5 in the case of the intact rail for frequencies up to the value $30 \mathrm{kHz}$.

This figure shows a frequency window around the frequency $12.5 \mathrm{kHz}$ that can be used to excite the rail. The analysis of the complex wave numbers showed that the attenuation increases strongly with frequency.

The knowledge of high-frequency attenuation is essential in order to identify the low-loss propagation modes that can be effectively used to operate detection.

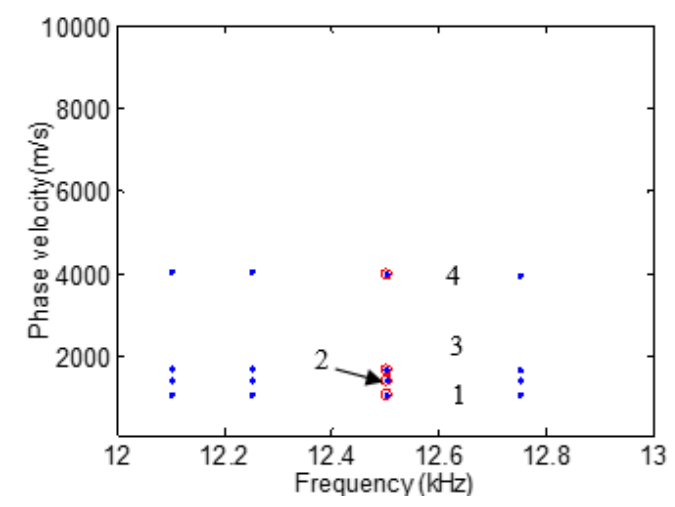

Figure 6: Zoom of dispersion curves in terms of phase velocity in $[12,13] \mathrm{kHz}$

Figure 6 shows a zoom on the dispersion curves in the frequency interval .The first four modes of the cross section are numbered from the low to the up.

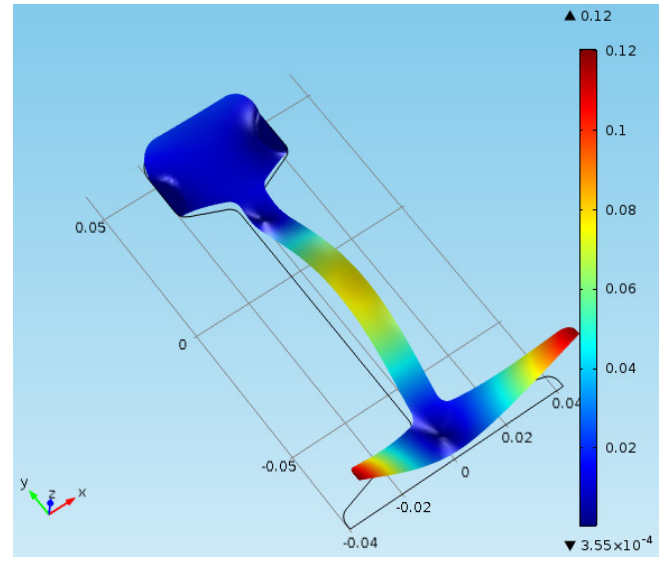

Figure 7: Intact rail; Displacement standard for $f=12.5 \mathrm{kHz}$ and $k=54.994 / \mathrm{m}$

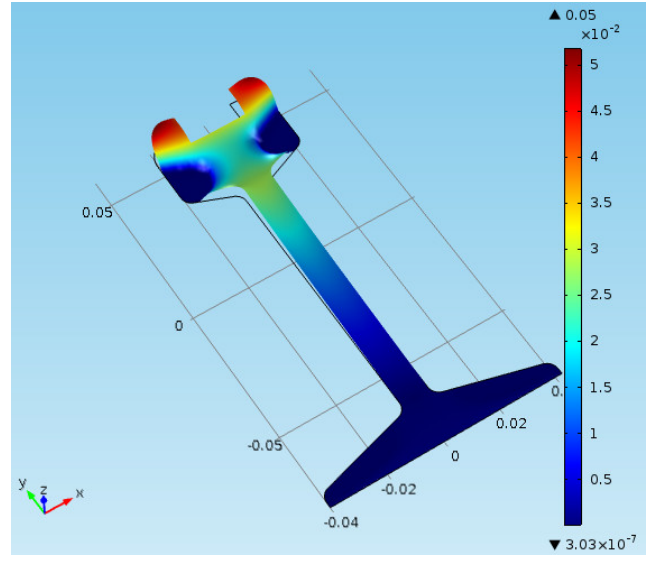

Figure 8: rail with defect; Displacement standard for $f=12.5 \mathrm{kHz}$ and $k=85.506 / \mathrm{m}$ 


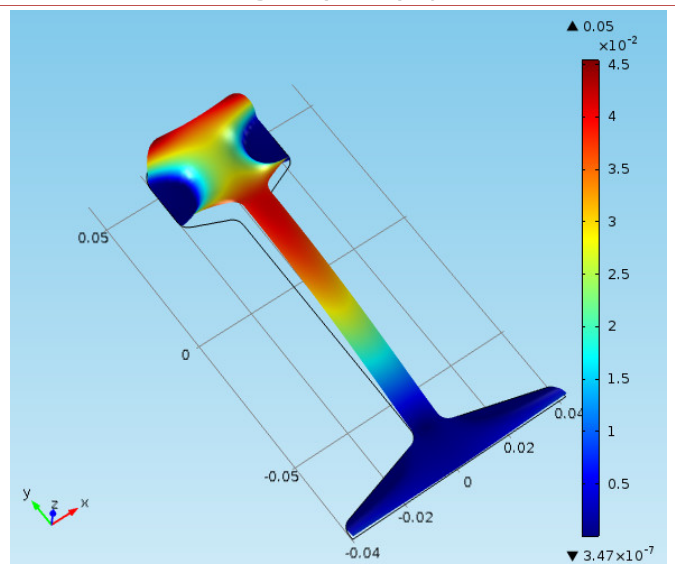

Figure 9: Intact rail; Displacement standard for $f=12.5 \mathrm{kHz}$ and $k=72.347 / \mathrm{m}$

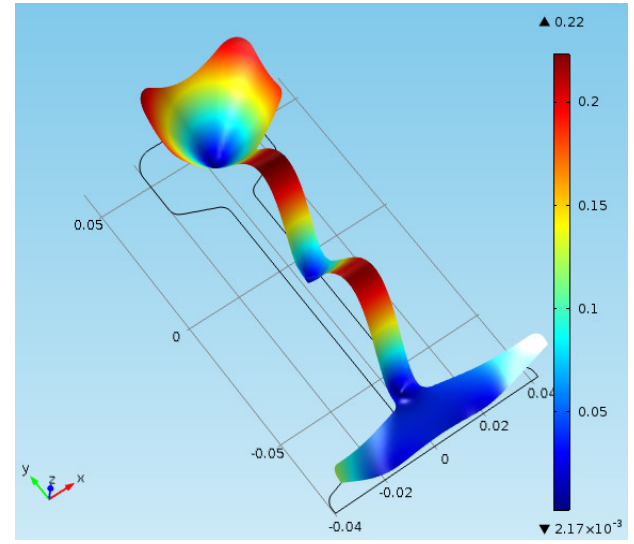

Figure 11: Intact rail; Displacement standard for $f=12.5 \mathrm{kHz}$ and $k=19.645 / \mathrm{m}$ $k=19.645 / m$

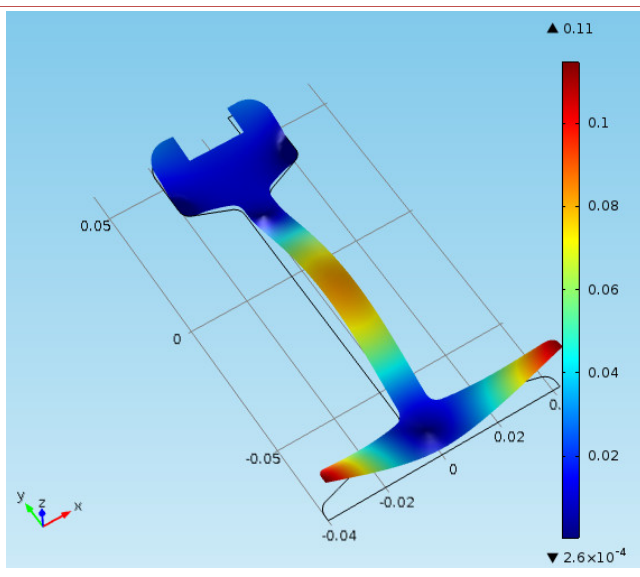

Figure 10: Rail with defect; Displacement standard for $f=12.5 \mathrm{kHz}$ and $k=55.513 / \mathrm{m}$

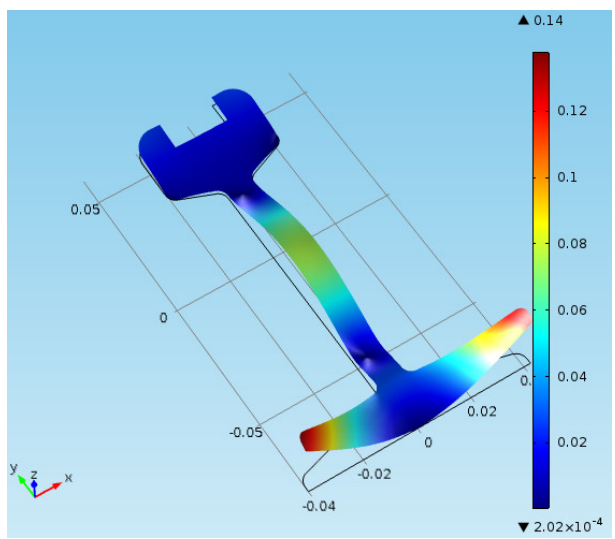

Figure 12: Rail with defect; Displacement standard for $f=12.5 \mathrm{kHz}$ and $k=47.025 / \mathrm{m}$

$$
k=19.645 / m
$$

This information can be exploited in practice in CND tests to target defects according to their position in the rail section.

\section{Conclusion}

The knowledge of these modes of propagation is very important in order to be able to design the ultrasonic wave excitation and detection system. This is to find the most suitable configuration for the type of defect.

\section{REFERENCES}

[1] B.D. Jeffery, M.L. Peterson, "Assessment of Rail Flaw Inspection Data," A Center of Excellence for Rural and Intermodal Transportation, 1999. 
Transactions on Machine Learning and Artificial Intelligence Vol 5 No 4, Aug 2017

[2] I. Bartoli, "Structural health monitoring by ultrasonic guided waves," PhD Thesis, University of California, San Diego, 2007.

[3] U. Zerbst, R. Lundén, K.O. Edel and R.A, Smith, "Introduction to the damage tolerance behaviour of railway rails," Engineering Fracture Mechanics,vol. 76, pp. 2563-2601, 2009.

[4] D.F. Cannon, K.O. Edel, S.L. Grassie, K. Sawley, "Rail defects: an overview," Fatigue and Fracture of Engineering Materials and Structures, pp. 865-887, 2003.

[5] K. Sawley, R. Reiff, "Rail Failure Assessment for the Office of the Rail Regulator," Report No P-00-070 produced by Transportation Technology Center Inc, Pueblo, Colorado, USA, 2000.

[6] C. Duverger, B. Prazil , "Un nouvel engin d'auscultation des rails à la SNCF," Revue Générale des Chemins de Fer. Paris: Bordas-Dunod,pp. 1-12, 1984.

[7] Y. Fan, S. Dixon, R.S.Edwards, X. Jian, "Ultrasonic surface wave propagation and interaction with surface defects on rail track head," NDT \& E International,pp. 71-7,2007.

[8] T. Hayashi, W. J. Song, J. L Rose, "Guided wave dispersion curves for a bar with an arbitrary cross-section, a rod and rail example," Ultrasonics,pp. 175-183,2003.

[9] COMSOL User's Guide and Documentation version 4.3a. COMSOL AB.

[10] M.V. Predoi, M. Castaings, B. Hosten, C. Bacon, "Wave propagation along transversely periodic structures," Journal of the Acoustical Society of America,pp. 1935-1944,2007.

[11] F.L. di Scalea., J. McNamara, "Ultrasonic NDE of railroad tracks: air-coupled cross-sectional inspection and long-range inspection," Insight - NDT\& Condition Monitoring, vol. 45,pp. 394-401, 2003. 